\title{
Lie Supergroups Obtained from 3-Dimensional Lie Superalgebras Associated to the Adjoint Representation and Having a 2-Dimensional Derived Ideal
}

\author{
I. Hernandez ${ }^{1}$ and R. Peniche ${ }^{2}$ \\ ${ }^{1}$ CIMAT, Apdo. Postal 402, C.P., 36000 Guanajuato, GTO, Mexico \\ ${ }^{2}$ Facultad de Matemáticas, Universidad Autónoma de Yucatán, Apdo. Postal 172, C.P., 97110 Mérida, \\ YUC, Mexico
}

Correspondence should be addressed to R. Peniche, pmena@uady.mx

Received 18 January 2007; Revised 23 May 2007; Accepted 31 October 2007

Recommended by Nils-Peter Skoruppa

We give the explicit multiplication law of the Lie supergroups for which the base manifold is a 3dimensional Lie group and whose underlying Lie superalgebra $\mathfrak{g}=\mathfrak{g}_{0} \oplus \mathfrak{g}_{1}$ which satisfies $\mathfrak{g}_{1}=\mathfrak{g}_{0}$, $\mathfrak{g}_{0}$ acts on $\mathfrak{g}_{1}$ via the adjoint representation and $\mathfrak{g}_{0}$ has a 2-dimensional derived ideal.

Copyright (c) 2008 I. Hernández and R. Peniche. This is an open access article distributed under the Creative Commons Attribution License, which permits unrestricted use, distribution, and reproduction in any medium, provided the original work is properly cited.

\section{Introduction}

A possible notion of superspace associated to a given 3-dimensional Lie algebra $\mathfrak{g}_{0}$ might be a Lie superalgebra $\mathfrak{g}=\mathfrak{g}_{0} \oplus \mathfrak{g}_{1}$. If one is to introduce a minimal set of assumptions, it seems quite natural to consider those superalgebras for which $\mathfrak{g}_{1}=\mathfrak{g}_{0}$ and for which the $\mathfrak{g}_{0}$ action on $\mathfrak{g}_{1}$ is the adjoint representation. A complete classification of the real and complex (3,3)-dimensional Lie superalgebras having precisely this restriction was recently obtained in [1]. The classification was given in terms of the dimension of the derived ideal $\mathfrak{g}_{0}^{\prime}=\left[\mathfrak{g}_{0}, \mathfrak{g}_{0}\right]$. The most trivial case was that corresponding to $\operatorname{dim} \mathfrak{g}_{0}^{\prime}=3$ whereas the most difficult case was that of $\operatorname{dim} \mathfrak{g}_{0}^{\prime}=0$. Our aim in this paper is to explicitly produce the real and complex Lie supergroups associated to the Lie superalgebras classified in [1] having a 2-dimensional derived ideal. This is just one step forward in our understanding of the real and complex $(3,3)$-dimensional Lie supergroups.

In order to maintain our exposition as self-contained as possible, we will summarize the basic statements from [1]. Once we fix the adjoint representation, it is well known (see [1,2]) that there are as many Lie superalgebras as bilinear symmetric maps $\Gamma: \mathfrak{g}_{0} \times \mathfrak{g}_{0} \rightarrow \mathfrak{g}_{0}$ satisfying

$$
\Gamma([x, y], z)+\Gamma(y,[x, z])=[x, \Gamma(y, z)] .
$$


Table 1: Full set of representatives of the isomorphism classes of the 3-dimensional Lie algebras $\mathfrak{g}_{0}$ that have 2-dimensional derived ideal. The classes were obtained from the canonical form of the $2 \times 2$ invertible matrix $A$. In the first two cases, the field $\mathbb{F}$ can be either $\mathbb{R}$ or $\mathbb{C}$.

\begin{tabular}{lcc}
\hline $\mathfrak{g}_{0}$ & $A$ & Constraints \\
\hline $\mathfrak{p}(\mathbb{F})$ & $\left(\begin{array}{ll}1 & 1 \\
0 & 1\end{array}\right)$ & - \\
$\mathfrak{q}_{\lambda}(\mathbb{F})$ & $\left(\begin{array}{ll}1 & 0 \\
0 & \lambda\end{array}\right)$ & $0<|\lambda| \leq 1$ \\
$\mathfrak{q}_{\lambda}^{1}(\mathbb{R})$ & $\left(\begin{array}{cc}\lambda & -1 \\
1 & 1\end{array}\right)$ & $\lambda \in \mathbb{R}$ \\
\hline
\end{tabular}

Furthermore, the isomorphism class of the Lie superalgebra defined by such a given $\Gamma$ is completely determined by its orbit under the action of the group $G \subset \operatorname{Aut}\left(\mathfrak{g}_{0}\right) \times \mathrm{GL}\left(\mathfrak{g}_{0}\right)$ of pairs $(T, S)$ satisfying $S \circ T^{-1} \circ \operatorname{ad}(\cdot)=\operatorname{ad}(\cdot) \circ S \circ T^{-1}$ given by

$$
\Gamma \longrightarrow(T, S) \cdot \Gamma=T\left(\Gamma\left(S^{-1}(\cdot), S^{-1}(\cdot)\right)\right) .
$$

Now, let $\mathbb{F}$ be either the real or the complex number field, and let $\mathfrak{g}_{0}$ be a fixed 3dimensional Lie algebra over $\mathbb{F}$. Let $\left\{e_{1}, e_{2}, e_{3}\right\}$ be a basis for $\mathfrak{g}_{0}$, and let $\operatorname{Sym}_{\mathrm{ad}}\left(\mathfrak{g}_{0}\right)$ be the real or complex vector space of symmetric, bilinear maps $\Gamma: \mathfrak{g}_{0} \times \mathfrak{g}_{0} \rightarrow \mathfrak{g}_{0}$ satisfying (1.1). Thus, we can identify the space $\operatorname{Sym}_{\mathrm{ad}}\left(\mathfrak{g}_{0}\right)$ with the set of triples $\left(\Gamma^{1}, \Gamma^{2}, \Gamma^{3}\right)$ of symmetric bilinear forms, for which $\Gamma(u, v)=\sum_{i=1}^{3} \Gamma^{i}(u, v) e_{i}$ satisfies (1.1).

Let $\mathfrak{g}_{0}^{\prime}=\left[\mathfrak{g}_{0}, \mathfrak{g}_{0}\right]$ be the derived ideal of $\mathfrak{g}_{0}$. Lie algebras having $\operatorname{dim} \mathfrak{g}_{0}^{\prime}=2$ can be classified by choosing $e_{1} \notin \mathfrak{g}_{0}^{\prime}$ and bringing ad $\left(e_{1}\right)$ into a convenient canonical form (see [3, 4] or [5] for details). Thus,

$$
\begin{aligned}
& {\left[e_{1}, e_{2}\right]=a e_{2}+c e_{3},} \\
& {\left[e_{1}, e_{3}\right]=b e_{2}+d e_{3}, \quad \text { so that }\left.\operatorname{ad}\left(e_{1}\right)\right|_{\mathfrak{g}_{0}^{\prime}} \longleftrightarrow A=\left(\begin{array}{ll}
a & b \\
c & d
\end{array}\right),} \\
& {\left[e_{2}, e_{3}\right]=0,}
\end{aligned}
$$

and, since $\operatorname{dim} \mathfrak{g}_{0}^{\prime}=2$, then $A$ must be an invertible matrix. Now, the classification up to isomorphism of the Lie algebras $\mathfrak{g}_{0}$ having $\operatorname{dim} \mathfrak{g}_{0}^{\prime}=2$ can be written as in Table 1 , where we followed the notation introduced in [1].

It is easy to see that the matrices of $\operatorname{ad}\left(e_{i}\right)$ take the forms

$$
\operatorname{ad}\left(e_{1}\right)=\left(\begin{array}{ll}
0 & 0 \\
0 & A
\end{array}\right), \quad \operatorname{ad}\left(e_{2}\right)=-\left(\begin{array}{cc}
0 & 0 \\
A \delta_{1} & 0
\end{array}\right), \quad \operatorname{ad}\left(e_{3}\right)=-\left(\begin{array}{cc}
0 & 0 \\
A \delta_{2} & 0
\end{array}\right),
$$

where $\delta_{1}=\left(\begin{array}{l}1 \\ 0\end{array}\right)$ and $\delta_{2}=\left(\begin{array}{l}0 \\ 1\end{array}\right)$. It is well known that the associated Lie groups for the Lie algebras we are dealing with here are the so-called unimodular groups $E(2)$ and $E(1,1)$ for $\mathfrak{q}_{1}(\mathbb{F})$ and $\mathfrak{q}_{-1}(\mathbb{F})$, respectively, and for the other Lie algebras, the associated Lie groups are called nonunimodular (see [6]). We shall denote by $G_{0}(A)$ any of those Lie groups.

It was proved in [1] that when $A$ is invertible, any triple $\left(\Gamma^{1}, \Gamma^{2}, \Gamma^{3}\right)$, for which $\Gamma(u, v)=$ $\sum_{i=1}^{3} \Gamma^{i}(u, v) e_{i}$ satisfies (1.1), is given, up to isomorphism, by

$$
\Gamma^{1}=p\left(\begin{array}{ccc}
1 & 0 & 0 \\
0 & 0 & 0 \\
0 & 0 & 0
\end{array}\right), \quad \Gamma^{2}=\frac{p}{2}\left(\begin{array}{lll}
0 & 1 & 0 \\
1 & 0 & 0 \\
0 & 0 & 0
\end{array}\right), \quad \Gamma^{3}=\frac{p}{2}\left(\begin{array}{lll}
0 & 0 & 1 \\
0 & 0 & 0 \\
1 & 0 & 0
\end{array}\right),
$$


where $p$ is an arbitrary element of the ground field $\mathbb{F}$ for the three classes $\mathfrak{p}(\mathbb{F}), \mathfrak{q}_{\lambda}(\mathbb{F})$, and $\mathfrak{q}_{\lambda}^{1}(\mathbb{R})$, with no relation between the parameters $p$ and $\lambda$.

What we do in this paper is to describe explicitly all the Lie supergroups whose underlying 3-dimensional Lie group is $G_{0}(A)$ and having as Lie superalgebras of left-invariant supervector fields the Lie superalgebras given by the triple $\left(\Gamma^{1}, \Gamma^{2}, \Gamma^{3}\right)$ above. The method is the one given in [7] and is essentially the one used in Lie's classical theory, that is, we give first a faithful representation of the Lie superalgebra into the Lie superalgebra of vector fields of some supermanifold, and obtain the local coordinate version of the supergroup multiplication law through composition of their integral flows depending on the integration parameters (see [7]).

In more detail, we aim to describe the multiplication law in terms of tetrads $(s, v ; \sigma, \theta)$, where $(s, v) \in \mathbb{F} \times \mathbb{F}^{2}$ are the local coordinates on the 3-dimensional Lie group $G_{0}(A)$, and $\sigma$ and $\theta$ are the odd coordinates on the supergroup $\left(G_{0}(A), \Lambda(E)\right)$, where $\Lambda(E)$ stands for the sheaf of sections of the exterior algebra bundle associated to the rank-3 vector bundle $E \rightarrow G_{0}(A)$, whose typical fiber $\mathfrak{g}_{0}$ can be decomposed as $S \oplus \mathfrak{g}_{0}^{\prime}$, with $S \simeq \mathfrak{g}_{0} / \mathfrak{g}_{0}^{\prime}$. Thus, $\sigma$ is a local section of $G_{0}(A) \times S \rightarrow G_{0}(A)$, and $\theta$ is a local section of $G_{0}(A) \times \mathfrak{g}_{0}^{\prime} \rightarrow G_{0}(A)$. Then, the product $\left(s^{\prime}, v^{\prime} ; \sigma^{\prime}, \theta^{\prime}\right) *(s, v ; \sigma, \theta)$ is given by (see Theorem 3.1)

$$
\left(s^{\prime}+s-\frac{p}{2} \sigma^{\prime} \sigma, v+e^{\left(-s+(p / 2) \sigma^{\prime} \sigma\right) A} v^{\prime}+\frac{p}{2} \sigma e^{-s A} \theta^{\prime} ; \sigma^{\prime}+\sigma, \theta+e^{\left(-s+(p / 2) \sigma^{\prime} \sigma\right) A} \theta^{\prime}+\sigma^{\prime} A v\right) .
$$

The point of giving such an explicit expression is to actually see the way odd coordinates are combined, within the supergroup composition law, to produce even sections. It was this interaction between even and odd coordinates what apparently had some physical and geometrical ideas that were worth studying but there are only a few explicit examples in the literature; some of them are incomplete, and some are relatively trivial.

Once we obtain the multiplication law for the different Lie supergroups, we give in Proposition 3.2 the supermorphisms that define the Lie supergroups within the spirit of $[7,8]$. Finally, in Proposition 3.3 we compute the left-invariant supervector fields associated to the Lie supergroups we have built, bringing us back to the Lie superalgebras we started with.

\section{Lie superalgebra representations}

Let us write

$$
A^{1}=\left(\begin{array}{cc}
0 & 0 \\
0 & A
\end{array}\right), \quad A^{2}=\left(\begin{array}{cc}
0 & 0 \\
\delta_{1} & 0
\end{array}\right), \quad A^{3}=\left(\begin{array}{cc}
0 & 0 \\
\delta_{2} & 0
\end{array}\right)
$$

where $\delta_{1}$ and $\delta_{2}$ are defined as above. Let $B^{r}$ and $C^{r}$ be $3 \times 3$ matrices $(r=1,2,3)$.

Proposition 2.1. Let $\mathfrak{g}=\mathfrak{g}_{0} \oplus \mathfrak{g}_{1}$ be a $(3,3)$-dimensional Lie superalgebra, where $\mathfrak{g}_{1}=\mathfrak{g}_{0}$, $\mathfrak{g}_{0}$ acts on $\mathfrak{g}_{1}$ via the adjoint representation, and $\operatorname{dim} \mathfrak{g}_{0}^{\prime}=2$. Let $\left\{e_{1}, e_{2}, e_{3}\right\}$ and $\left\{f_{1}, f_{2}, f_{3}\right\}$ be bases for $\mathfrak{g}_{0}$ and $\mathfrak{g}_{1}$, respectively. Let $V=V_{0} \oplus V_{1}$ be a $(3,3)$-dimensional supervector space and let $\rho: \mathfrak{g} \rightarrow \mathfrak{g l}(V)$ be a linear map such that

$$
\rho\left(e_{i}\right)=\left(\begin{array}{cc}
A^{i} & 0 \\
0 & A^{i}
\end{array}\right), \quad \rho\left(f_{i}\right)=\left(\begin{array}{cc}
0 & B^{i} \\
C^{i} & 0
\end{array}\right) .
$$


Then, the choices

$$
B^{i}=\frac{p}{2} A^{i}, \quad C^{i}=K A^{i}
$$

where $K=\left(\begin{array}{cc}0 & 0 \\ 0 & A^{-1}\end{array}\right)$, turn $\rho: \mathfrak{g} \rightarrow \mathfrak{g l}(V)$ into a faithful representation of the Lie superalgebra $\mathfrak{g}$.

Proof. Let us write

$$
B^{r}=\left(\begin{array}{cc}
\beta_{r} & u_{r}^{T} \\
v_{r} & B_{r}
\end{array}\right), \quad C^{r}=\left(\begin{array}{cc}
\epsilon_{r} & x_{r}^{T} \\
y_{r} & C_{r}
\end{array}\right)
$$

where $\beta_{r}, \epsilon_{r} \in \mathbb{F}, u_{r}, v_{r}, x_{r}, y_{r} \in \mathbb{F}^{2}$ and $B_{r}$ and $C_{r}$ are $2 \times 2$ matrices.

From the definition of $\rho\left(e_{i}\right)$, it is straightforward to check that $\left\{\rho\left(e_{i}\right)\right\}$ defines a Lie algebra isomorphic to that described in (1.3).

In order to have $\mathfrak{g}_{0}$ acting on $\mathfrak{g}_{1}$ via the adjoint representation, we must have

$$
\left[\rho\left(e_{i}\right), \rho\left(f_{j}\right)\right]= \begin{cases}a \rho\left(f_{2}\right)+c \rho\left(f_{3}\right), & i=1, j=2 \\ b \rho\left(f_{2}\right)+d \rho\left(f_{3}\right), & i=1, j=3 \\ -a \rho\left(f_{2}\right)-c \rho\left(f_{3}\right), & i=2, j=1 \\ -b \rho\left(f_{2}\right)-d \rho\left(f_{3}\right), & i=3, j=1\end{cases}
$$

but $\left[\rho\left(e_{i}\right), \rho\left(f_{j}\right)\right]=\left(\begin{array}{cc}0 & A^{i} B^{j}-B^{j} A^{i} \\ A^{i} C^{j}-C^{j} A^{i} & 0\end{array}\right)$, so we obtain

$$
\begin{aligned}
& B^{1}=\left(\begin{array}{cc}
\beta_{1} & 0 \\
0 & B_{1}
\end{array}\right), \quad B^{2}=\left(\begin{array}{cc}
0 & 0 \\
v_{2} & 0
\end{array}\right), \quad B^{3}=\left(\begin{array}{cc}
0 & 0 \\
v_{3} & 0
\end{array}\right), \\
& C^{1}=\left(\begin{array}{cc}
\epsilon_{1} & 0 \\
0 & C_{1}
\end{array}\right), \quad C^{2}=\left(\begin{array}{cc}
0 & 0 \\
y_{2} & 0
\end{array}\right), \quad C^{3}=\left(\begin{array}{cc}
0 & 0 \\
y_{3} & 0
\end{array}\right) \text {, }
\end{aligned}
$$

whereand 1 is the $2 \times 2$ identity matrix. Now, we have to satisfy the condition $\left[\rho\left(f_{i}\right), \rho\left(f_{j}\right)\right]=$ $\sum_{k} \Gamma_{i j}^{k} \rho\left(e_{k}\right)$, but $\left[\rho\left(f_{i}\right), \rho\left(f_{j}\right)\right]=\left(\begin{array}{cc}B^{i} C^{j}+B^{j} C^{i} & 0 \\ 0 & C^{i} B^{j}+C^{j} B^{i}\end{array}\right)$. Then,

$$
\begin{gathered}
\beta_{1} \epsilon_{1}=0, \quad 2 B_{1} C_{1}=p A=2 C_{1} B_{1} \\
v_{2} \epsilon_{1}+B_{1} y_{2}=\frac{p}{2} \delta_{1}=y_{2} \beta_{1}+C_{1} v_{2}, \quad v_{3} \epsilon_{1}+B_{1} y_{3}=\frac{p}{2} \delta_{2}=y_{3} \beta_{1}+C_{1} v_{3} .
\end{gathered}
$$


With no loss of generality, we can choose $\epsilon_{1}=0$ and $y_{2}=A^{-1} \delta_{1}, y_{3}=A^{-1} \delta_{2}$, so that $C_{1}=1$ and $B_{1}=(p / 2) A$. Finally, by choosing $v_{2}=(p / 2) \delta_{1}-\beta_{1} y_{2}$ and $v_{3}=(p / 2) \delta_{2}-\beta_{1} y_{3}$ we find that all equations are satisfied. Actually, we can always choose $\beta_{1}=0$, obtaining the expressions given in the statement.

The choices we have made in the proof of Proposition 2.1 produce easier exponential matrices (see Theorem 3.1 below). Since the representation $\rho$ is to be faithful, different choices made in the proof would have produced Lie superalgebras isomorphic to $\mathfrak{g}$ inside $\mathfrak{g l}(V)$. Therefore, the corresponding supergroups obtained via the constructive method used in Theorem 3.1 would have been isomorphic at the end. This is so because Lie's theory looks for faithfully realizing $\mathfrak{g}$ in terms of vector fields whose integral flows will eventually define the supergroup multiplication law via composition of (local) diffeomorphisms.

\section{Lie supergroups for which $A$ is invertible}

Once we have the different Lie superalgebras represented in $\mathfrak{g l}(V)$ for some $(3,3)$ dimensional supervector space $V$, we proceed to find a supermanifold that actually carries a Lie supergroup structure following essentially the same steps followed in the classical theory of Lie. In fact, we can always obtain explicitly a Lie group structure for $G_{0}(A)$ from its Lie algebra $\mathfrak{g}_{0}$, where $\operatorname{Lie}\left(G_{0}(A)\right)=\mathfrak{g}_{0}$. So, let us write $(s, v)$ as the local coordinates described in the introduction.

Theorem 3.1. Let $\mathfrak{g}=\mathfrak{g}_{0} \oplus \mathfrak{g}_{1}$ be a Lie superalgebra satisfying $\mathfrak{g}_{1}=\mathfrak{g}_{0}$, $\mathfrak{g}_{0}$ acting on $\mathfrak{g}_{1}$ via the adjoint representation, and having 2-dimensional derived ideal $\mathfrak{g}_{0}^{\prime}=\left[\mathfrak{g}_{0}, \mathfrak{g}_{0}\right]$. The Lie supergroups whose underlying Lie superalgebras are $\mathfrak{g}$ have the following multiplication law for the products of $\left(s^{\prime}, v^{\prime} ; \sigma^{\prime}, \theta^{\prime}\right)$ and $(s, v ; \sigma, \theta)$ :

$$
\left(s^{\prime}+s-\frac{p}{2} \sigma^{\prime} \sigma, v+e^{\left(-s+(p / 2) \sigma^{\prime} \sigma\right) A} v^{\prime}+\frac{p}{2} \sigma e^{-s A} \theta^{\prime} ; \sigma^{\prime}+\sigma, \theta+e^{\left(-s+(p / 2) \sigma^{\prime} \sigma\right) A} \theta^{\prime}+\sigma^{\prime} A v\right) .
$$

Proof. According to $[7,8]$ we only have to compute the exponential of the matrices $\rho\left(e_{i}\right)$ and $\rho\left(f_{j}\right)$ given in Proposition 2.1, and the supergroup composition law will be obtained from first principles using the ODE theory in supermanifolds and following Lie's original techniques as described before (see [7]). If we denote by $\left(t_{1}, t_{2}, t_{3} ; \tau_{1}, \tau_{2}, \tau_{3}\right)$ the composition

$$
\operatorname{Exp}\left(t_{1} \rho\left(e_{1}\right)\right) \circ \operatorname{Exp}\left(t_{2} \rho\left(e_{2}\right)\right) \circ \operatorname{Exp}\left(t_{3} \rho\left(e_{3}\right)\right) \circ \operatorname{Exp}\left(\tau_{1} \rho\left(f_{1}\right)\right) \circ \operatorname{Exp}\left(\tau_{2} \rho\left(f_{2}\right)\right) \circ \operatorname{Exp}\left(\tau_{3} \rho\left(f_{3}\right)\right),
$$

we notice that $\left(t_{1}, t_{2}, t_{3} ; \tau_{1}, \tau_{2}, \tau_{3}\right)=\left(\begin{array}{cc}P & Q \\ -R & P\end{array}\right)$, where

In order to find the multiplication law, we have to find when the following identity holds:

$$
\left(t_{1}^{\prime \prime}, t_{2}^{\prime \prime}, t_{3}^{\prime \prime} ; \tau_{1}^{\prime \prime}, \tau_{2}^{\prime \prime}, \tau_{3}^{\prime \prime}\right)=\left(t_{1}^{\prime}, t_{2}^{\prime}, t_{3}^{\prime} ; \tau_{1}^{\prime}, \tau_{2}^{\prime}, \tau_{3}^{\prime}\right) \cdot\left(t_{1}, t_{2}, t_{3} ; \tau_{1}, \tau_{2}, \tau_{3}\right),
$$


that is, we have to solve

$$
\left(\begin{array}{cc}
P^{\prime \prime} & Q^{\prime \prime} \\
-R^{\prime \prime} & P^{\prime \prime}
\end{array}\right)=\left(\begin{array}{cc}
P^{\prime} & Q^{\prime} \\
-R^{\prime} & P^{\prime}
\end{array}\right)\left(\begin{array}{cc}
P & Q \\
-R & P
\end{array}\right)=\left(\begin{array}{cc}
P^{\prime} P-Q^{\prime} R & P^{\prime} Q+Q^{\prime} P \\
-\left(R^{\prime} P+P^{\prime} R\right) & P^{\prime} P-R^{\prime} Q
\end{array}\right)
$$

From $P^{\prime \prime}=P^{\prime} P-Q^{\prime} R$, we obtain

$$
\begin{aligned}
& e^{t_{1}^{\prime \prime} A}=e^{\left(t_{1}^{\prime}+t_{1}\right) A}\left(1-\frac{p}{2} \tau_{1}^{\prime} \tau_{1} A\right)=e^{\left(t_{1}^{\prime}+t_{1}-(p / 2) \tau_{1}^{\prime} \tau_{1}\right) A}, \\
& e^{t_{1}^{\prime \prime} A}\left(\left(\begin{array}{c}
t_{2}^{\prime \prime} \\
t_{3}^{\prime \prime}
\end{array}\right)-\frac{p}{2} \tau_{1}^{\prime \prime}\left(\begin{array}{c}
\tau_{2}^{\prime \prime} \\
\tau_{3}^{\prime \prime}
\end{array}\right)\right) \\
& =e^{t_{1}^{\prime} A}\left(\left(\begin{array}{c}
t_{2}^{\prime} \\
t_{3}^{\prime}
\end{array}\right)-\frac{p}{2} \tau_{1}^{\prime}\left(\begin{array}{c}
\tau_{2}^{\prime} \\
\tau_{3}^{\prime}
\end{array}\right)\right)+e^{\left(t_{1}^{\prime}+t_{1}\right) A}\left(\left(\begin{array}{c}
t_{2} \\
t_{3}
\end{array}\right)-\frac{p}{2} \tau_{1}\left(\begin{array}{c}
\tau_{2} \\
\tau_{3}
\end{array}\right)-\frac{p}{2} \tau_{1}^{\prime}\left(\begin{array}{c}
\tau_{2} \\
\tau_{3}
\end{array}\right)\right) ;
\end{aligned}
$$

and from $R^{\prime \prime}=R^{\prime} P+P^{\prime} R$, we obtain

$$
\begin{aligned}
& \tau_{1}^{\prime \prime} e^{t_{1}^{\prime \prime} A}=\left(\tau_{1}^{\prime}+\tau_{1}\right) e^{\left(t_{1}^{\prime}+t_{1}\right) A}, \\
& e^{t_{1}^{\prime \prime} A} A^{-1}\left(\begin{array}{c}
\tau_{2}^{\prime \prime} \\
\tau_{3}^{\prime \prime}
\end{array}\right) \\
& =e^{t_{1}^{\prime} A} A^{-1}\left(\begin{array}{c}
\tau_{2}^{\prime} \\
\tau_{3}^{\prime}
\end{array}\right)+e^{\left(t_{1}^{\prime}+t_{1}\right) A} \cdot A^{-1}\left(\begin{array}{c}
\tau_{2} \\
\tau_{3}
\end{array}\right)+\tau_{1}^{\prime} e^{\left(t_{1}^{\prime}+t_{1}\right) A}\left(\left(\begin{array}{c}
t_{2} \\
t_{3}
\end{array}\right)-\frac{p}{2} \tau_{1}\left(\begin{array}{c}
\tau_{2} \\
\tau_{3}
\end{array}\right)\right) .
\end{aligned}
$$

Now, it is straightforward to prove that

$$
\begin{gathered}
t_{1}^{\prime \prime}=t_{1}+t_{1}^{\prime}-\frac{p}{2} \tau_{1}^{\prime} \tau_{1} \\
\left(\begin{array}{c}
t_{2}^{\prime \prime} \\
t_{3}^{\prime \prime}
\end{array}\right)=\left(\begin{array}{c}
t_{2} \\
t_{3}
\end{array}\right)++e^{\left(-t_{1}+(p / 2) \tau_{1}^{\prime} \tau_{1}\right) A}\left(\begin{array}{c}
t_{2}^{\prime} \\
t_{3}^{\prime}
\end{array}\right)+\frac{p}{2} \tau_{1} e^{-t_{1} A}\left(\begin{array}{c}
\tau_{2}^{\prime} \\
\tau_{3}^{\prime}
\end{array}\right) \\
\tau_{1}^{\prime \prime}=\tau_{1}+\tau_{1}^{\prime} \\
\left(\begin{array}{c}
t_{2}^{\prime \prime} \\
t_{3}^{\prime \prime}
\end{array}\right)=\left(\begin{array}{c}
\tau_{2} \\
\tau_{3}
\end{array}\right)+e^{\left(-t_{1}+(p / 2) \tau_{1}^{\prime} \tau_{1}\right) A}\left(\begin{array}{c}
\tau_{2}^{\prime} \\
\tau_{3}^{\prime}
\end{array}\right)+\tau_{1}^{\prime} A\left(\begin{array}{c}
t_{2} \\
t_{3}
\end{array}\right)
\end{gathered}
$$

and defining $s=t_{1}, v=\left(\begin{array}{c}t_{2} \\ t_{3}\end{array}\right), \sigma=\tau_{1}$, and $\theta=\left(\begin{array}{c}t_{2} \\ t_{3}\end{array}\right)$, we find the multiplication law given in the statement. 
From (3.7), we can write the multiplication law in terms of morphisms as in $[7,8]$.

Proposition 3.2. Let $\mathcal{G}(p, A)$ be the $(3,3)$-dimensional supermanifold whose underlying Lie group is $G_{0}(A)$ and let $\left\{x_{1}, x_{2}, x_{3} ; \xi_{1}, \xi_{2}, \xi_{3}\right\}$ be local coordinates. For $i=1,2$, let $\pi_{i}: \mathcal{G}(p, A) \times \mathcal{G}(p, A) \rightarrow$ $\mathcal{G}(p, A)$ be the direct product projections. Then $\mathcal{G}(p, A)$ is a Lie supergroup endowed with the morphism $m: \mathcal{G}(p, A) \times \mathcal{G}(p, A) \rightarrow \mathcal{G}(p, A)$ defined by

$$
\begin{gathered}
m^{*}\left(x_{1}\right)=\pi_{2}^{*}\left(x_{1}\right)+\pi_{1}^{*}\left(x_{1}\right)-\frac{p}{2} \pi_{1}^{*}\left(\xi_{1}\right) \pi_{2}^{*}\left(\xi_{1}\right), \\
\left(\begin{array}{l}
m^{*}\left(x_{2}\right) \\
m^{*}\left(x_{3}\right)
\end{array}\right)=\left(\begin{array}{c}
\pi_{2}^{*}\left(x_{2}\right) \\
\pi_{2}^{*}\left(x_{3}\right)
\end{array}\right)+e^{\left(-\pi_{2}^{*}\left(x_{1}\right)+(p / 2) \pi_{1}^{*}\left(\xi_{1}\right) \pi_{2}^{*}\left(\xi_{1}\right)\right) A}\left(\begin{array}{c}
\pi_{1}^{*}\left(x_{2}\right) \\
\pi_{1}^{*}\left(x_{3}\right)
\end{array}\right)+\frac{p}{2} \pi_{2}^{*}\left(\xi_{1}\right) e^{\left(-\pi_{2}^{*}\left(x_{1}\right) A\right.}\left(\begin{array}{c}
\pi_{1}^{*}\left(\xi_{2}\right) \\
\pi_{1}^{*}\left(\xi_{3}\right)
\end{array}\right), \\
m^{*}\left(\xi_{1}\right)=\pi_{2}^{*}\left(\xi_{1}\right)+\pi_{1}^{*}\left(\xi_{1}\right), \\
\left(\begin{array}{l}
m^{*}\left(\xi_{2}\right) \\
m^{*}\left(\xi_{3}\right)
\end{array}\right)=\left(\begin{array}{c}
\pi_{2}^{*}\left(\xi_{2}\right) \\
\pi_{2}^{*}\left(\xi_{3}\right)
\end{array}\right)+e^{\left(-\pi_{2}^{*}\left(x_{1}\right)+(p / 2) \pi_{1}^{*}\left(\xi_{1}\right) \pi_{2}^{*}\left(\xi_{1}\right)\right) A}\left(\begin{array}{c}
\pi_{1}^{*}\left(\xi_{2}\right) \\
\pi_{1}^{*}\left(\xi_{3}\right)
\end{array}\right)+\pi_{1}^{*}\left(\xi_{1}\right) A\left(\begin{array}{c}
\pi_{2}^{*}\left(x_{2}\right) \\
\pi_{2}^{*}\left(x_{3}\right)
\end{array}\right) ;
\end{gathered}
$$

the morphism $\varepsilon: \mathcal{G}(p, A) \rightarrow \mathcal{G}(p, A)$ defined by $\varepsilon^{*}\left(x_{i}\right)=\varepsilon^{*}\left(\xi_{i}\right)=0$, and the morphism $i: \mathcal{G}(p, A) \rightarrow$ $\mathcal{G}(p, A)$ defined by

$$
\begin{gathered}
i^{*}\left(x_{1}\right)=-x_{1}, \\
\left(\begin{array}{c}
i^{*}\left(x_{2}\right) \\
m^{*}\left(x_{3}\right)
\end{array}\right)=-e^{x_{1} A}\left(\begin{array}{c}
x_{2}-\frac{p}{2} \xi_{1} \xi_{2} \\
x_{3}-\frac{p}{2} \xi_{1} \xi_{3}
\end{array}\right), \\
i^{*}\left(\xi_{1}\right)=-\xi_{1} \\
\left(\begin{array}{c}
i^{*}\left(\xi_{2}\right) \\
m^{*}\left(\xi_{3}\right)
\end{array}\right)=-e^{x_{1} A}\left(\begin{array}{c}
\xi_{2} \\
\xi_{3}
\end{array}\right)+\xi_{1} A e^{x_{1} A}\left(\begin{array}{l}
x_{2} \\
x_{3}
\end{array}\right) .
\end{gathered}
$$

Proof. It is straightforward to check that

$$
\begin{gathered}
\left(\pi_{1}, m \circ\left(\pi_{2}^{*}, \pi_{3}^{*}\right)\right)^{*} \circ m^{*}=\left(m \circ\left(\pi_{1}^{*}, \pi_{2}^{*}\right), \pi_{3}^{*}\right)^{*} \circ m^{*}, \\
(\varepsilon, i d)^{*} \circ m^{*}=i d^{*}=(i d, \varepsilon)^{*} \circ m^{*}, \\
(i, i d)^{*} \circ m^{*}=\varepsilon^{*}=(i d, i)^{*} \circ m^{*},
\end{gathered}
$$

which are the associative law, the identity element, and inverse element properties,hold. 
Proposition 3.3. Assuming the hypotheses of Proposition 3.2, the left-invariant supervector fields can be written as $X=\lambda_{1} X_{1}+\lambda_{2} X_{2}+\lambda_{3} X_{3}+\mu_{1} Y_{1}+\mu_{2} Y_{2}+\mu_{3} Y_{3}$, where

$$
\begin{aligned}
X_{1}= & \frac{\partial}{\partial x_{1}}-\left(a x_{2}+b x_{3}\right) \frac{\partial}{\partial x_{2}}-\left(c x_{2}+d x_{3}\right) \frac{\partial}{\partial x_{3}}-\left(a \xi_{2}+b \xi_{3}\right) \frac{\partial}{\partial \xi_{2}}-\left(c \xi_{2}+d \xi_{3}\right) \frac{\partial}{\partial \xi_{3}} \\
X_{2}= & \frac{\partial}{\partial x_{2}}+a \xi_{1} \frac{\partial}{\partial \xi_{2}}+c \xi_{1} \frac{\partial}{\partial \xi_{3}} \\
X_{3}= & \frac{\partial}{\partial x_{3}}+b \xi_{1} \frac{\partial}{\partial \xi_{2}}+d \xi_{1} \frac{\partial}{\partial \xi_{3}} \\
Y_{1}= & \frac{\partial}{\partial \xi_{1}}+\frac{p}{2}\left[\left(a \xi_{2}+b \xi_{3}\right) \xi_{1} \frac{\partial}{\partial \xi_{2}}+\left(c \xi_{2}+d \xi_{3}\right) \xi_{1} \frac{\partial}{\partial \xi_{3}}\right] \\
& +\frac{p}{2}\left[\xi_{1} \frac{\partial}{\partial x_{1}}+\left(\xi_{2}-\left(a x_{2}+b x_{3}\right) \xi_{1}\right) \frac{\partial}{\partial x_{2}}+\left(\xi_{3}-\left(c x_{2}+d x_{3}\right) \xi_{1}\right) \frac{\partial}{\partial x_{3}}\right] \\
Y_{2}= & \frac{\partial}{\partial \xi_{2}} \\
Y_{3}= & \frac{\partial}{\partial \xi_{3}}
\end{aligned}
$$

and $\lambda_{i}, \mu_{j} \in \mathbb{F}$. Furthermore, the Lie superalgebra defined by the left-invariant supervector fields $\left\{X_{1}, X_{2}, X_{3}, Y_{1}, Y_{2}, Y_{3}\right\}$ is isomorphic to the Lie superalgebra given by (1.3) and (1.5).

Proof. Any supervector field can be written as $X=\sum f_{i}\left(\partial / \partial x_{i}\right)+g_{i}\left(\partial / \partial \xi_{i}\right)$ and $X$ is a leftinvariant supervector field if the supervector field

$$
\widehat{X}=\sum \pi_{2}^{*}\left(f_{i}\right) \frac{\partial}{\partial \pi_{2}^{*}\left(x_{i}\right)}+\pi_{2}^{*}\left(g_{i}\right) \frac{\partial}{\partial \pi_{2}^{*}\left(\xi_{i}\right)}
$$

satisfies

$$
\varepsilon^{(2)} \circ \widehat{X} \circ\left(p_{1}, m\right)^{*}=\varepsilon^{2} \circ\left(p_{1}, m\right)^{*} \circ \widehat{X}
$$

where $\varepsilon^{(2)}: \mathcal{G}(p, A) \rightarrow \mathcal{G}(p, A) \times \mathcal{G}(p, A)$ is given by $\varepsilon^{(2)^{*}} \circ p_{1}^{*}=i d^{*}$ and $\varepsilon^{(2)^{*}} \circ p_{2}^{*}=\varepsilon^{*}$, as in [7]. By Proposition 3.2 we have the explicit multiplication morphisms $m^{*}$ and $\varepsilon^{*}$ and applying the local coordinates $\left\{\pi_{1}^{*}\left(x_{i}\right), \pi_{2}^{*}\left(x_{i}\right), \pi_{1}^{*}\left(\xi_{i}\right), \pi_{2}^{*}\left(\xi_{i}\right)\right\}$ on both sides of (3.13) we found the restrictions for $f_{i}$ 's and $g_{i}$ 's and they are written as in the statement.

Finally, in order to prove that $\left\{X_{1}, X_{2}, X_{3}, Y_{1}, Y_{2}, Y_{3}\right\}$ defines the Lie superalgebra given in the beginning, we just have to compute the Lie superbrackets given for the supervector fields, namely, $[X, Z]=X \circ Z-(-1)^{|X||Z|} Z \circ X$ to check that it is precisely the same Lie superalgebra defined by (1.3) and (1.5). By defining the correspondence

$$
e_{i} \mapsto X_{i}, \quad f_{j} \mapsto Y_{j}
$$

we conclude that they are isomorphic. 


\section{Acknowledgments}

The authors would like to acknowledge with thanks the partial support received from the following grants: CONACYT Grant 46274, and Programa del Mejoramiento del Profesorado, Secretara de Educación, Grant PROMEP/103.5/2526 PTC-45-D. They would like to thank Professor Adolfo Sánchez-Valenzuela for enlightening discussions during the genesis of this work, and the kind hospitality received by the authors at CIMAT and Facultad de Matemáticas, at UADY. Last but not least, they would like to thank the referees for their comments, criticism, and recommendations contributed to clear various passages of the original manuscript.

\section{References}

[1] I. Hernández, G. Salgado, and O. A. Sánchez-Valenzuela, "Lie superalgebras based on a 3-dimensional real or complex Lie algebra," Journal of Lie Theory, vol. 16, no. 3, pp. 539-560, 2006.

[2] M. Scheunert, The Theory of Lie Superalgebras: An Introduction, vol. 716 of Lecture Notes in Mathematics, Springer, Berlin, Germany, 1979.

[3] W. Fulton and J. Harris, Representation Theory: A First Course, vol. 129 of Graduate Texts in Mathematics, Springer, New York, NY, USA, 1991.

[4] N. Jacobson, Lie Algebras, Republication of the 1962 Original, Dover, New York, NY, USA, 1979.

[5] A. L. Onishchik and Ė. B. Vinberg, Lie Groups and Algebraic Groups, Springer Series in Soviet Mathematics, Springer, Berlin, Germany, 1990.

[6] J. Milnor, "Curvatures of left invariant metrics on Lie groups," Advances in Mathematics, vol. 21, no. 3, pp. 293-329, 1976.

[7] R. Peniche and O. A. Sánchez-Valenzuela, "Lie supergroups supported over $\mathrm{GL}_{2}$ and $U_{2}$ associated to the adjoint representation," Journal of Geometry and Physics, vol. 56, no. 6, pp. 999-1028, 2006.

[8] V. S. Varadarajan, Supersymmetry for Mathematicians: An Introduction, vol. 11 of Courant Lecture Notes in Mathematics, American Mathematical Society, New York, NY, USA, 2004. 


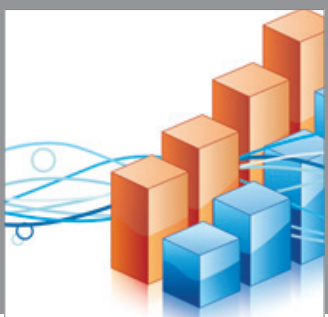

Advances in

Operations Research

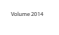

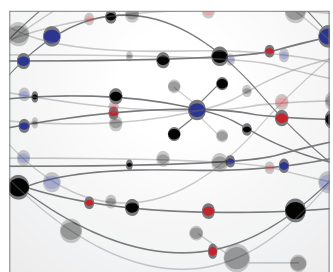

\section{The Scientific} World Journal
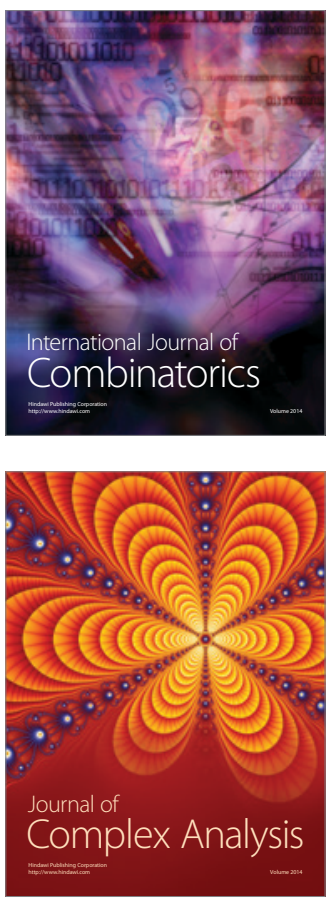

International Journal of

Mathematics and

Mathematical

Sciences
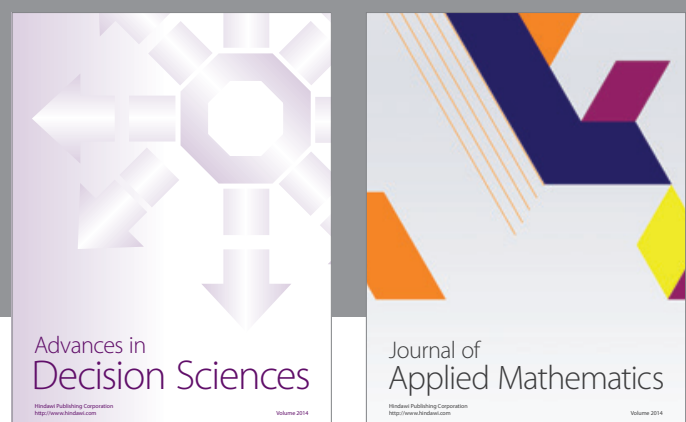

Journal of

Applied Mathematics
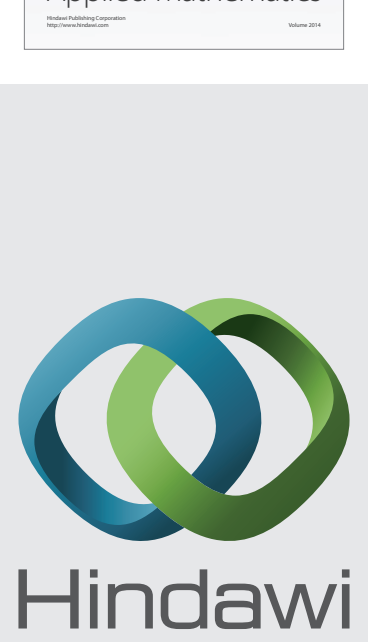

Submit your manuscripts at http://www.hindawi.com
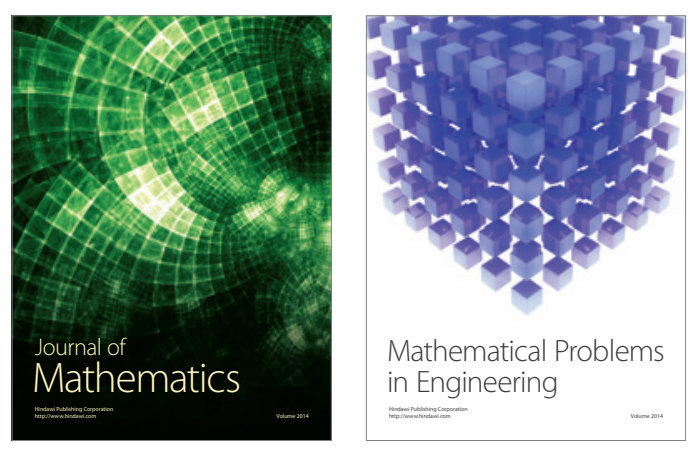

Mathematical Problems in Engineering
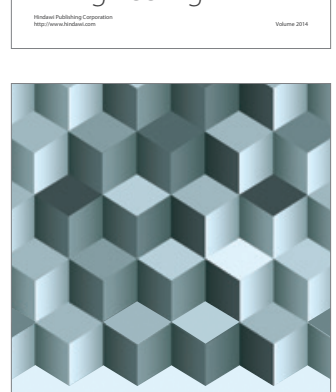

Journal of

Function Spaces
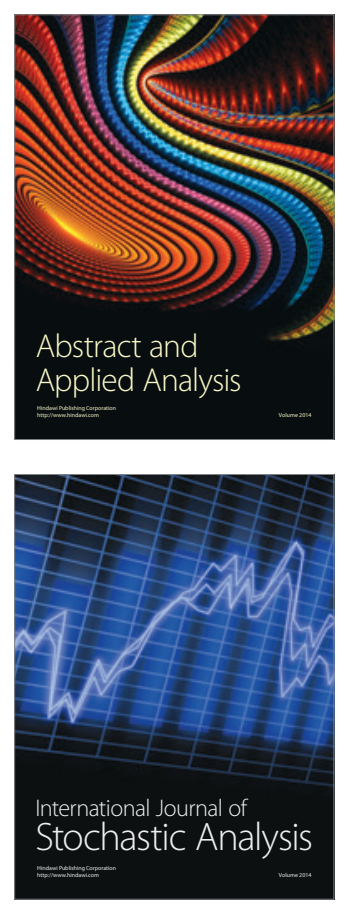

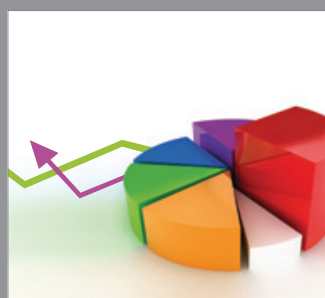

ournal of

Probability and Statistics

Promensencen
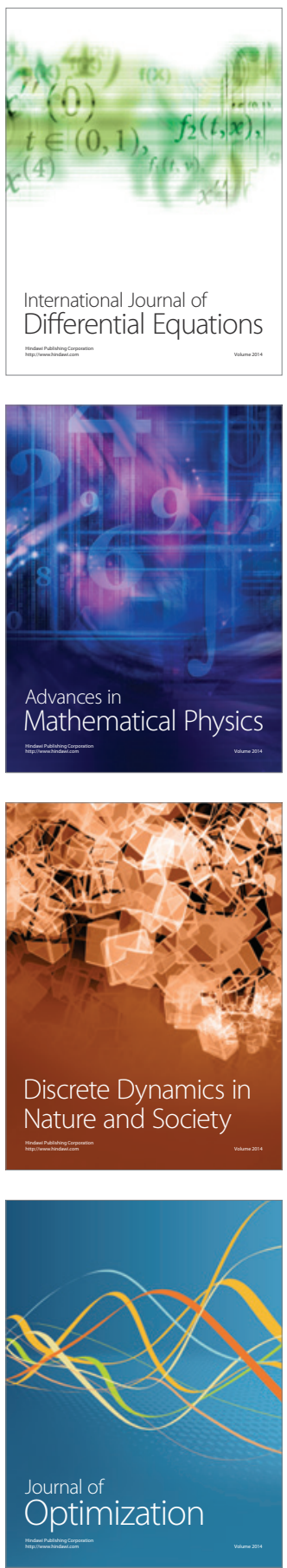